\title{
Expression, purification and identification of an immunogenic fragment in the ectodomain of prostate-specific membrane antigen
}

\author{
RONG TAO, ZHENHUA NI, CHONG LIU, MIN ZHU, XIAOWEN JI, \\ XUEMIN CHEN, JIANGFAN SHEN and SHAOHUA TU
}

Department of Nuclear Medicine, Putuo Hospital, Shanghai University of Traditional Chinese Medicine, Shanghai 200062, P.R. China

Received April 3, 2015; Accepted November 25, 2015

DOI: $10.3892 /$ etm.2016.3005

\begin{abstract}
The present study aimed to identify, express and purify an immunogenic fragment in the ectodomain of prostate-specific membrane antigen (PSMA) within a fusion protein. The PSMA amino acid sequence published in National Center for Biotechnology Information GenBank was used to determine sequence homology and immunogenic index analyses, additionally using BLASTN, Protean and ExPASy software to predict the polypeptide sequences of immunogenic epitopes. The gene sequence encoding the ectodomain of the polypeptide immunogenic fragments, containing the identified immunogenic epitopes, was generated using whole-gene synthesis. Prokaryotic expression vector pET-32a-r-ectodomain-PSMA was constructed and the recombinant plasmids were transformed into competent BL21 (DE3) Escherichia coli, which was followed by induction of recombinant protein expression using isopropyl- $\beta$-D-thiogalactopyranoside. Fusion proteins were isolated and purified using affinity chromatography and their immune activity was subsequently investigated using western blot analysis. Purified protein was used to immunize BALB/c mice in order to generate polyclonal antibodies, and the binding of polyclonal antibodies to prostate cancer cell lines in vitro was evaluated using flow cytometry. A total of 3 polypeptide fragments with high specificity were identified following analysis using numerous software packages, and the gene sequences encoding regions containing the 2 most immunogenic fragments were synthesized and successfully inserted into the prokaryotic expression vector pET-32a-r-ectodomain-PSMA. The recombinant PSMA protein fragment had a molecular weight of $\sim 50 \mathrm{kDa}$ and $95 \%$ purity. Western blot analysis revealed that the r-ectodomain-PSMA fusion protein specifically bound to the anti-PSMA ectodomain monoclonal antibody.
\end{abstract}

Correspondence to: Mr. Shaohua Tu, Department of Nuclear Medicine, Putuo Hospital, Shanghai University of Chinese Traditional Medicine, 409 Meiling North Road, Shanghai 200062, P.R. China

E-mail: tush_99@163.com

Key words: prostate-specific membrane antigen, major epitope region, antigen purification, prokaryotic expression
Flow cytometry demonstrated that polyclonal antibodies raised against these recombinant proteins could specifically bind to PSMA-positive LNCaP cells, but not to PSMA-negative PC-3 cells. An immunogenic fragment in the ectodomain of PSMA was successfully expressed and purified. The present study, therefore, provides a basis for the preparation of an anti-PSMA small humanized monoclonal antibody.

\section{Introduction}

Prostate cancer presents with malignant tumors that threaten the health of middle-aged and elderly men. Concomitant with a global increase in the age of the population, the incidence of prostate cancer has risen (1). No effective method for the early diagnosis of this disease currently exists. The decision to undergo surgical resection of prostate tumors depends on the incidence of prostate cancer metastases; the clinical diagnosis of metastases typically relies on bone scans, but these cannot detect metastases in the sentinel lymph nodes or soft tissue (2). However, human-derived antibody preparation, together with radioimmunoimaging technology have emerged as potential molecular tools for the early detection and diagnosis of prostate cancer metastases $(3,4)$.

Prostate-specific membrane antigen (PSMA) is a type II transmembrane glycoprotein located in the prostate epithelial cells, and is comprised of 750 amino acid residues across 3 domains; these are an intracellular domain of 19 amino acids, a transmembrane domain of 24 amino acids and an ectodomain of 707 amino acids. PSMA is a more sensitive and specific marker of prostate tumors compared with prostate-specific antigen (4,5), as it is highly expressed in prostate cancer, particularly androgen-independent and metastatic prostate cancer, but is rarely expressed in normal non-prostate tissue. PSMA has high tissue specificity, making it an ideal target protein for the diagnosis and treatment of prostate cancer (6); however, it also has an N-terminal transmembrane domain with marked hydrophobicity, making it unsuitable for use as an immunogen. To the best of our knowledge, no study of PSMA ectodomain immunogenic sites has previously been reported. As prokaryotically-expressed PSMA protein fragments contain linear protein molecules that are not folded in physiologically-appropriate three-dimensional conformations, the immunogenicity of these linear protein molecules is unclear. In addition, to the best of our knowledge, there 
have been no previous reports on the binding specificity of prepared anti-PSMA. These unknowns have hindered the study of the molecular diagnostic imaging and radioimmunotherapy in the diagnosis and treatment of prostate cancer. The present study used bioinformatics to predict the immunogenicity of fragments of PSMA ectodomain polypeptide, and the immune binding activity of these prokaryotically-expressed fragments was characterized.

\section{Materials and methods}

Plasmids and strains. pET-32a (cat. no. 69015; Novagen, Inc., Madison, WI, USA) plasmids acted as vectors. DH5 $\alpha$ and BL21 (DE3) pLysS strains (cat. no. CB106; Tiangen Biotech Co., Ltd., Beijing, China) of competent Escherichia coli (E. coli) were used for inducible protein expression.

Cells. LNCaP (androgen-sensitive human prostate adenocarcinoma) and PC-3 (human prostate adenocarcinoma) cell lines (Shanghai Institute of Biochemistry and Cell Biology, Chinese Academy of Science, Shanghai, China) were used for verification of polyclonal antibody binding.

Experimental animals. A total of three 6-8-week-old BALB/c male mice (Shanghai Institute of Materia Medica, Chinese Academy of Science, Shanghai, China), weighing 18-20 g, were maintained under specific pathogen-free conditions in the present study. The protocol of the present study was approved by the Institutional Animal Care and Use Committee of Shanghai University of Traditional Chinese Medicine (Shanghai, China).

Reagents. Reagents used were sourced as follows: Restriction endonucleases KpnI and XhoI, RPMI-1650, fetal bovine serum (Thermo Fisher Scientific, Inc., Waltham, MA, USA); T4 ligase, isopropyl- $\beta$-D-thiogalactopyranoside (IPTG), a $10 \mathrm{~kb}$ DNA ladder (cat. no. B6000241; Sangon Biotech Co., Ltd., Shanghai, China) a protein marker (cat. no. SM0431; Thermo Fisher Scientific, Inc.), an EZNA plasmid extraction kit (cat. no. D6943; Omega Bio-Tek Inc., Norcross, GA, USA); a plasmid purification kit (Merck Sharpe \& Dohme, Shanghai, China); acrylamide and methylene bis-acrylamide (Genview Scientific Inc., El Monte, CA, USA); a 3,000-unit dialysis bag, sodium dodecyl sulfate (SDS) and Tris base (Sino-American Biotechnology Co., Ltd., Shanghai, China); anti-PSMA (YPSMA-1) mouse monoclonal antibody (cat. no. ab19071, Abcam, Shanghai, China); and Freund's complete adjuvant and fluorescein isothiocyanate (FITC)-labeled goat anti-mouse polyclonal immunoglobulin G (cat. no. F9006; Sigma-Aldrich, St. Louis, MO, USA). All reagents were of analytical grade.

Prediction of the PSMA ectodomain polypeptide antigen. Using the primary structure of PSMA reported in National Center for Biotechnology Information GenBank (AAA60209.1), the hydrophilicity, antigen indices and homology of PSMA ectodomain amino acid sequences were analyzed using ExPASy, Protean (http://www.expasy.org/) and BLASTN software (https://blast.ncbi.nlm.nih.gov/) (7). Amino acid sequences with high hydrophilicity and antigen indices, and low homology were selected as polypeptide antigen sequences; specific immunogenic peptides were then selected by determining the most appropriate polypeptide antigen sequence and, thus, expression region of PSMA to be used. The selected polypeptide immunogenic fragment is referred to as the recombinant PSMA antigen ectodomain fragment (r-ectodomain-PSMA).

Synthesis of the PSMA antigen ectodomain fragment-encoding gene. Using the PSMA gene sequence (GenBank M9948.1) and the prokaryotic expression host, the nucleotide sequence of the PSMA ectodomain was optimized to account for preferred codon usage in the expression host (7). The nucleotide sequence was generated by Sangon Biotech Co., Ltd. The synthesized gene fragment was then inserted into the pET-32a vector at restriction enzyme sites KpnI and XhoI.

Construction of prokaryotic expression vector. Double restriction endonuclease digestion using KpnI and XhoI was performed in the target gene and the pET-32a expression vector; this was followed by digested product purification using TIANgel Extraction kit (cat. no. DP209; Tiangen Biotech Co., Ltd.). The digested target fragment and plasmid were ligated at $16^{\circ} \mathrm{C}$ for $4 \mathrm{~h}$ using T4 DNA ligase (cat. no. RT406; Tiangen Biotech Co., Ltd.) and transformed into a DH5 $\alpha$ bacterial strain by a heat shock pulse at $42^{\circ} \mathrm{C}$ for $90 \mathrm{~s}$. The successfully transformed plasmid colonies growing on the agar plate were selected and individually inoculated into fresh culture tubes, which were incubated at $37^{\circ} \mathrm{C}$ in a $220 \mathrm{rpm}$ shaker overnight. This was followed by plasmid extraction using a EZNA plasmid extraction kit. KpnI and XhoI double restriction endonuclease digestions and recombinant plasmid sequencing were used to confirm target gene insertion.

Inducible expression of recombinant proteins. pET-32a-r-ectodomain-PSMA plasmid was transformed into E. coli BL21 (DE3) pLysS. A single colony of positive pET-32a-r-ectodomain-PSMA/E c coli BL21 (DE3) pLysS was inoculated into $5 \mathrm{ml} \mathrm{LB}$ medium containing ampicillin $(100 \mu \mathrm{g} / \mathrm{ml})$ and incubated overnight at $37^{\circ} \mathrm{C}$ in a $220-\mathrm{rpm}$ shaker. The overnight culture was inoculated into fresh LB medium ( $1 \%$ volume/volume) containing a final concentration of $100 \mu \mathrm{g} / \mathrm{ml}$ ampicillin and incubated at $37^{\circ} \mathrm{C}$ at $220 \mathrm{rpm}$ until the culture reached an optical density at $600 \mathrm{~nm}$ of $0.6-0.8$; this was followed by addition of IPTG solution, at a final concentration of $0.5 \mathrm{mM}$, to induce protein expression. A number of incubation temperatures and durations $\left(15\right.$ or $25^{\circ} \mathrm{C}$ overnight or $37^{\circ} \mathrm{C}$ for $5 \mathrm{~h}$ ) of recombinant protein expression induction were evaluated. Subsequent to induction, recombinant protein expression was terminated by centrifuging the solution at $15,294 \mathrm{x} \mathrm{g}$, at $4^{\circ} \mathrm{C}$ for $5 \mathrm{~min}$ to collect the cells. The harvested cells were then resuspended in phosphate-buffered saline (PBS) and lysed by ultrasonication, and the lysate was centrifuged at $20,817 \mathrm{xg}$, at $4^{\circ} \mathrm{C}$ for $30 \mathrm{~min}$. The supernatant and pellet of the lysate were analyzed using 10\% SDS-polyacrylamide gel electrophoresis (PAGE).

Purification using affinity chromatography. Nickel ion affinity chromatography was performed in accordance with a previous study by Liu et al (8). PBS at pH 7.0 was used to wash the bacterial culture pellet. PBS $(10 \mathrm{ml})$ and an additional $10 \mathrm{ml}$ PBS containing $8 \mathrm{~mol} / \mathrm{l}$ urea were then added to resuspend the bacteria, which was followed by ultrasonication and centrifugation at $20,817 \times \mathrm{g}, 4^{\circ} \mathrm{C}$ for $30 \mathrm{~min}$. The 
Table I. Predicted polypeptide immunogens.

\begin{tabular}{llccc}
\hline Polypeptide immunogen & Amino acid sequence & Sequence beginning & Sequence end & Length, amino acids \\
\hline Polypeptide immunogen 1 & ESWTKKSPSPEFSGM & 495 & 509 & 15 \\
Polypeptide immunogen 2 & APSSHNKYAGESFPG & 693 & 707 & 15 \\
Polypeptide immunogen 3 & KMGGSAPPDSSWRGS & 308 & 322 & 15 \\
\hline
\end{tabular}

pellet was dissolved in binding buffer in a chromatography cabinet overnight. Dissolved pellet was then centrifuged at $20,817 \times \mathrm{g}, 4^{\circ} \mathrm{C}$ for $30 \mathrm{~min}$ to collect the supernatant. The supernatant was subsequently used for affinity chromatography, following filtration through a $0.45 \mu \mathrm{m}$ microporous membrane. Eluted proteins were collected and dialysed successfully using a 3,000-unit dialysis bag. The purity of the target protein was evaluated using SDS-PAGE.

Western blot analysis. 10\% SDS-PAGE was used to separate the proteins, which were then transferred to a $0.45-\mu \mathrm{m}$ polyvinylidene fluoride (PVDF) membrane using a semi-dry transfer device at $12 \mathrm{~V}$ for $40 \mathrm{~min}$. The PVDF membrane was then blocked in Tris-buffered saline with $0.1 \%$ Tween 20 (TBS-T), containing $5 \%$ bovine serum albumin (BSA; cat. no. ST023; Beyotime Institute of Biotechnology, Haimen, China), at $4^{\circ} \mathrm{C}$ overnight. Subsequent to being washed with TBS-T 4 times (10 min each), the membrane was incubated with a 1:500 dilution of YPSMA-1 (in 5\% BSA-containing TBS-T) at room temperature for $2 \mathrm{~h}$. Subsequent to additional TBS-T washes, a 1:10,000 dilution of alkaline phosphatase-labeled goat anti-mouse IgG (diluted in TBS-T containing 5\% BSA) was incubated with the membrane at room temperature for 2 h. A 5-bromo-4-chloro-3-indolyl phosphate/nitro blue tetrazolium chloride kit (cat. no. C3206; Beyotime Institute of Biotechnology) was used for the colorimetric development of the PVDF membrane following additional washing steps.

Preparation of the polyclonal antibody. A mixture of $0.5 \mathrm{ml}$ purified recombinant protein $(250 \mu \mathrm{g})$ and an equal volume of Freund's complete adjuvant were used to immunize 3 male BALB/c mice at 6-8 weeks of age. Each mouse was immunized subcutaneously at three sites on the back with $15 \mu \mathrm{g}$ protein in each injection site, 4 times at 20-day intervals, followed by an intraperitoneally-administered booster dose (5th dose) containing $23 \mu \mathrm{g}$ protein. Serum from each animal was collected 2 weeks after this immunization for measurement of the titer using an indirect enzyme-linked immunosorbent assay (9).

Flow cytometry. The prostate cancer LNCaP cell line, expressing PSMA, and the PC-3 cell line, not expressing PSMA, were cultured as described in a previous study (10). LNCaP and PC-3 cells were harvested during the logarithmic growth phase in order to prepare live cell suspensions. Subsequent to being washed in PBS, the cell suspension was diluted to $1 \times 10^{9}$ cells/ 1 in PBS; $100 \mu 1$ of cell suspension from each cell line was then collected and incubated with a 1:2,500 dilution of recombinant PSMA mouse polyclonal antibodies at room temperature for $30 \mathrm{~min}$. Cells were washed 3 times

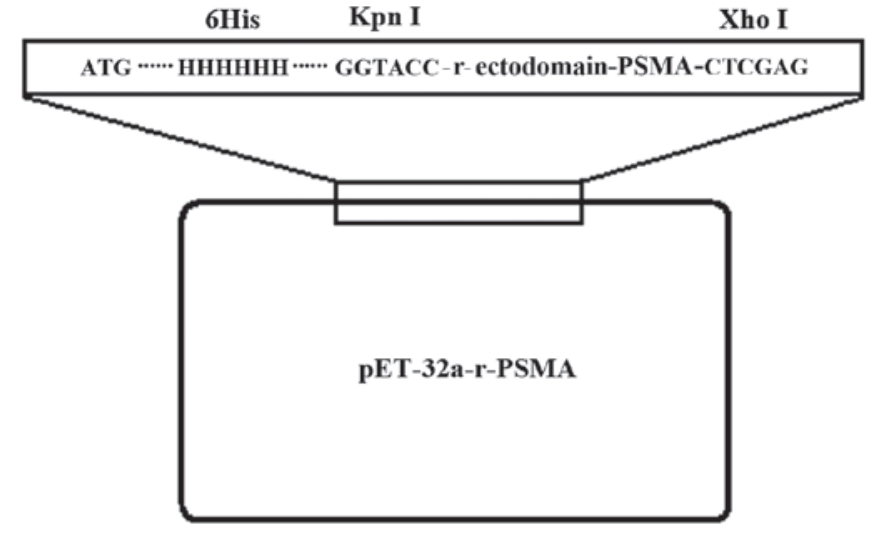

Figure 1. Diagram of the recombinant plasmid. PSMA, prostate-specific membrane antigen.

with PBS and centrifuged at $500 \mathrm{x} \mathrm{g}$ for $5 \mathrm{~min}$ at room temperature prior to incubation with FITC-labeled goat anti-mouse $\mathrm{IgG}$ at room temperature for a further $30 \mathrm{~min}$, in the dark. The cells were washed 3 subsequent times in PBS and centrifuged. Following this, $0.5 \mathrm{ml}$ PBS buffer was added to resuspend the cells prior to measurements and flow cytometry analyses.

Statistical analysis. Statistical analyses were performed using SPSS 15.0 software (SPSS, Inc., Chicago, IL, USA). Data were expressed as the mean \pm standard deviation. Differences were analyzed for significance using a two-tailed Student's t-test for independent means. $\mathrm{P}<0.05$ was considered to indicate a statistically significant difference.

\section{Results}

Prediction of the PSMA ectodomain polypeptide immunogen. BLASTN, Protean and ExPASy software was used to determine the hydrophilicity, immunogenic indices and amino acid homology of 707 amino acids of the PSMA ectodomain. Table I demonstrates the 3 immunogenic polypeptide sequences selected for high hydrophilicity and immunogen indices, and low amino acid homology. The polypeptide immunogens 1 and 2, which had higher priority scores and were in the C-terminal region of PSMA were therefore selected. The expression region containing polypeptide immunogen 1 and 2 is referred to as the recombinant PSMA ectodomain polypeptide immunogenic fragment, and this contained a total of 310 amino acids (amino acids 440-750).

Construction of the expression vector. Using the whole gene sequence of PSMA, the polypeptide immunogenic 


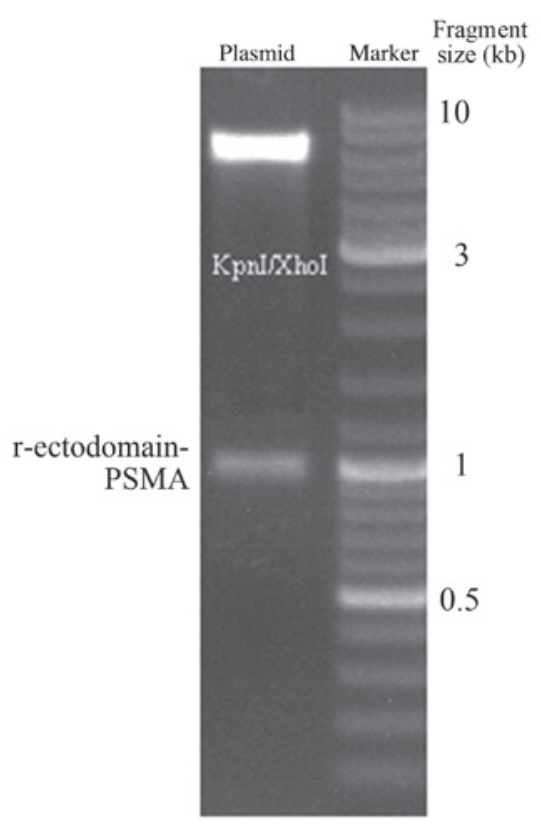

Figure 2. Gel electrophoresis of recombinant plasmid following $K p n \mathrm{I}$ and XhoI double enzymatic digestion. PSMA, prostate-specific membrane antigen.

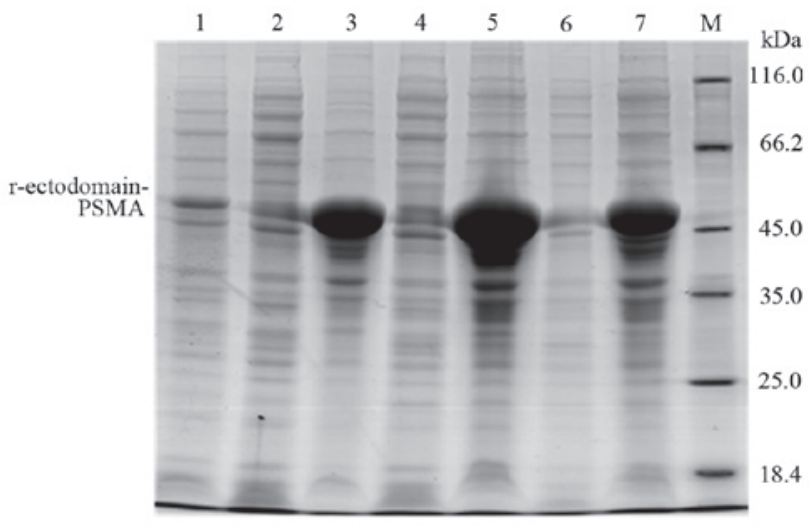

Figure 3. Protein gel demonstrating prokaryotic expression of recombinant protein. 1 , uninduced control; $2,15^{\circ} \mathrm{C}$ overnight induction (supernatant); $3,15^{\circ} \mathrm{C}$ overnight induction (pellet); $4,25^{\circ} \mathrm{C}$ overnight induction (supernatant); $5,25^{\circ} \mathrm{C}$ overnight induction (pellet); $6,37^{\circ} \mathrm{C} 5 \mathrm{~h}$ induction (supernatant); $7,37^{\circ} \mathrm{C} 5 \mathrm{~h}$ induction (pellet); M, marker. PSMA, prostate-specific membrane antigen.

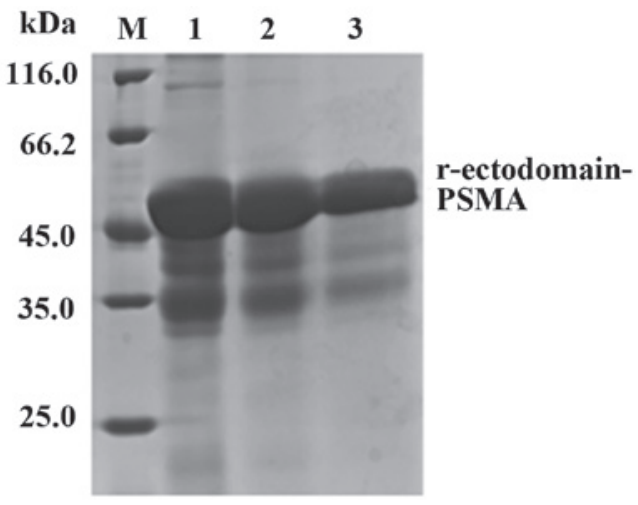

Figure 4. Recombinant protein purified by affinity chromatography. M, marker; 1, $10 \mathrm{mM}$ imidazole; 2, $20 \mathrm{mM}$ imidazole; 3, $500 \mathrm{mM}$ imidazole. PSMA, prostate-specific membrane antigen.

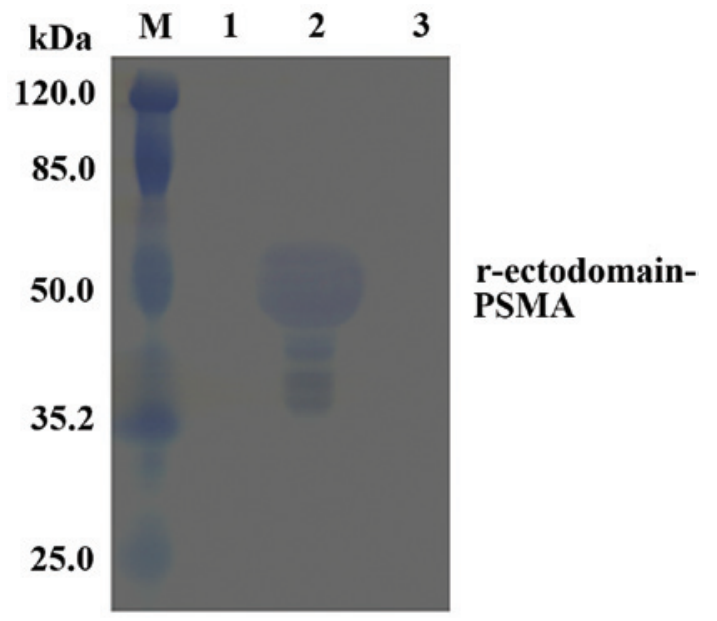

Figure 5. Western blot analysis of the recombinant protein. M, marker; 1 , supernatant; 2 , pellet; 3 , negative control. PSMA, prostate-specific membrane antigen.

A

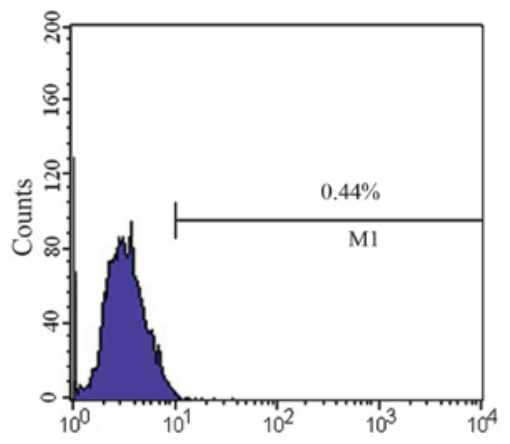

B

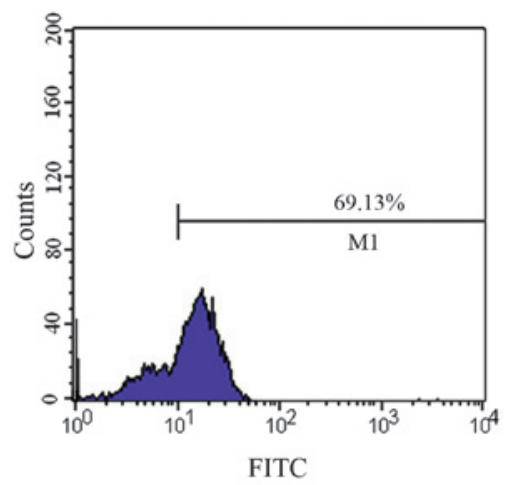

Figure 6. Flow cytometry. (A) Recombinant polyclonal antibody and PC-3 cells; (B) recombinant polyclonal antibody and LNCaP cells. FITC, fluorescein isothiocyanate.

fragment sequence was optimized to account for preferred codon usage, and this was followed by oligonucleotide synthesis. The synthesized target DNA fragment was ligated into the pET-32a vector in order to construct the expression vector pET-32a-r-ectodomain-PSMA (Fig. 1). The pET-32a-r-ectodomain-PSMA was then digested by restriction endonuclease $K p n \mathrm{I}$ and $\mathrm{XhoI}$. Gel electrophoresis revealed that the cleavage of $K p n I$ and $X h o I$ generated the target fragment, which was consistent with the expected fragment size of $930 \mathrm{bp}$ (Fig. 2). The endonuclease cleavage fragment was verified by sequencing, and the results of 
this were consistent with the PSMA sequence, indicating successful construction of the expression vector.

Expression of the r-ectodomain-PSMA recombinant protein. Subsequent to transforming the pET-32a-r-ectodomain-PSMA construct into $E$. coli BL21 (DE3) pLysS, protein expression was induced using $0.5 \mathrm{mM}$ IPTG under varying conditions. Upon terminating the expression, 10\% SDS-PAGE was used to verify differing protein expression. The target protein was expressed in the pellet at $15^{\circ} \mathrm{C}, 25^{\circ} \mathrm{C}$ and $37^{\circ} \mathrm{C}$ (Fig. 3). The most abundant protein expression was induced by $0.5 \mathrm{mM}$ IPTG overnight at $25^{\circ} \mathrm{C}$.

Purification of the r-ectodomain-PSMA recombinant protein. Nickel ion affinity chromatography was conducted using marker protein $6 \mathrm{His}$ to purify the target protein and collect imidazole-eluted protein. SDS-PAGE revealed that the size of the purified target protein matched its predicted size of $50 \mathrm{kDa}$ (Fig. 4), with $295 \%$ purity.

Western blot analysis of the r-ectodomain-PSMA recombinant protein. Anti-YPSMA-1 ectodomain monoclonal antibody was used in western blot analysis to assess the expressed r-ectodomain-PSMA recombinant protein. The fusion protein was demonstrated to specifically bind the anti-PSMA ectodomain monoclonal antibody (Fig. 5).

Verification of binding activity of polyclonal antibodies using flow cytometry. Flow cytometry (Fig. 6) revealed that the binding rate of PSMA-positive $\mathrm{LNCaP}$ cells to the recombinant protein polyclonal antibody was $69.1 \%$, whilst the binding rate of PSMA-negative PC-3 to the recombinant protein polyclonal antibody was $0.44 \%$ (basically no binding).

\section{Discussion}

Antibody recognition of an antigen does not involve recognition of the whole molecule but rather the immunogenic epitopes (11). For immunogenic recognition, an immunogen does not necessarily have to express the full-length protein; 300-400 amino acids in the N-terminal and C-terminal regions of proteins are frequently recognized as immunogenic fragments. In accordance with this, the present study predicted 3 PSMA immunogenic fragments with high immunogenicity (Table I). Peptides in the N-terminal and C-terminal regions are preferable to peptides in the middle of a protein for immunogen selection, as peptides in the middle of a protein are more likely to reside inside the protein upon folding; in the present study, polypeptide immunogen 3 was eliminated for this reason. The entire protein was also too large to be suitable for prokaryotic expression. However, the length of the C-terminal region containing the predicted polypeptide immunogens 1 and 2 is between 300-400 amino acids, and for this reason, a fragment of 310 amino acids at the C-terminal of PSMA ectodomain (amino acids 440-750) was selected for prokaryotic expression.

Whole sequence synthesis of the 310 amino acids of the C-terminal region was performed by ligating the peptide into a pET-32a vector, thereby constructing the expression vector of pET-32a-r-ectodomain-PSMA. Subsequent to the restriction endonuclease KpnI and XhoI cleavage of pET-32a-r-ectodomain-PSMA, the size of the target fragment was verified using gel electrophoresis; this was confirmed to be consistent with the expected fragment size of $930 \mathrm{bp}$, indicating a successful construction of the recombinant plasmid. As demonstrated by Fig. 3, the optimal conditions for recombinant protein induction were overnight and at $25^{\circ} \mathrm{C}$. Following nickel ion affinity chromatography, SDS-PAGE analysis revealed that the purified protein size $(\sim 50 \mathrm{kDa})$ was consistent with the predicted value.

The present study used YPSMA-1 ectodomain monoclonal antibody to verify the target protein expression. The binding site of this antibody was within the 716-723 amino acids of the PSMA ectodomain (12). In the current study, the expressed PSMA ectodomain protein fragment also contained the amino acids of the antibody-binding site. Application of YPSMA-1 in western blot analysis revealed the expression of recombinant protein as a clear $50-\mathrm{kDa}$ protein band (Fig. 5), indicating immunogenic activity of the prepared recombinant protein. Furthermore, recombinant protein addition combined with adjuvant immunization in BALB/c mice produced a high-titer polyclonal antibody. Flow cytometry confirmed that this antibody could bind to PSMA-positive LNCaP cells, but not to PSMA-negative PC-3 cells (Fig. 6), indicating that the polyclonal antibodies prepared using the immunogenic fragments produced a specific immune response.

The present study demonstrated that the anti-PSMA ectodomain monoclonal antibody has considerable value in the diagnosis of prostate cancer in radioimmunoimaging. However, the penetrability and clearance of large monoclonal antibodies is generally poor in vivo. Repeated use of murine monoclonal antibodies can lead to human anti-mouse antibody reactions, limiting clinical applications (13-15). For this reason, small humanized antibodies have emerged as possible diagnostic tools, and the PSMA ectodomain recombinant protein generated in the current study provides a basis for screening and production of small humanized antibodies.

\section{References}

1. Yu SQ and Xia SJ: Development of molecular targeted treatment for prostate cancer. Zhong Hua Yi Xue Za Zhi Bian Ji Bu 87: 718-719, 2007 (In Chinese).

2. Ross JS, Gray KE, Webb IJ, Gray GS, Rolfe M, Schenkein DP, Nanus DM, Millowsky MI and Bander NH: Antibody-based therapeutics: Focus on prostate cancer. Cancer Metastasis Rev 24: 521-537, 2005.

3. Zhou LL and Wang XS: Overview of prostate-specific membrane antigen. An Hui Hua Gong Bian Ji Bu 35: 28-31, 34, 2010 (In Chinese).

4. You J, Cozzi P, Walsh B, Willcox M, Kearsley J, Russell P and $\mathrm{Li} \mathrm{Y}$ : Innovative biomarkers for prostate cancer early diagnosis and progression. Crit Rev Oncol Hematol 73: 10-22, 2010.

5. Perner S, Hofer MD, Kim R, Shah RB, Li H, Möller P, Hautmann RE, Gschwend JE, Kuefer R and Rubin MA: Prostate-specific membrane antigen expression as a predictor of prostate cancer progression. Hum Pathol 38: 696-701, 2007.

6. Mhawech-Fauceglia P, Zhang S, Terracciano L, Sauter G, Chadhuri A, Herrmann FR and Penetrante R: Prostate-specific membrane antigen (PSMA) protein expression in normal and neoplastic tissues and its sensitivity and specificity in prostate adenocarcinoma: An immunohistochemical study using mutiple tumour tissue microarray technique. Histopathology 50: 472-483, 2007. 
7. Xiao Z, Jiang X, Beckett ML and Wright GL Jr: Generation of a baculovirus recombinant prostate-specific membrane antigen and its use in the development of a novel protein biochip quantitative immunoassay. Protein Expr Purif 19: 12-21, 2000.

8. Liu T, Toriyabe Y and Berkman CE: Purification of prostate-specific membrane antigen using conformational epitope-specific antibody-affinity chromatography. Protein Expr Purif 49: 251-255, 2006.

9. Zuo X, Liu C, Tang G and Jin Y: Mouse PD-1 gene fragment expression of extracellular domain, purification, and its polyclonal antibody preparation. Zhong Guo Mian Yi Xue Za Zhi Bian Ji Bu 10: 919-922, 2010 (In Chinese).

10. Tu SH, Shen JF, Tao R, Ji XW and Wang YC: The preparation of 99Tcm-J591 and its imaging of nude mice bearing human prostate cancer. Ji Chu Yi Xue Yu Lin Chuang 33: 284-288, 2013 (In Chinese).

11. Liu H, Liu B, He X and Zhao XT: Expression, purification, and identification the major epitope region of herpes simplex virus type 1 glycoprotein D. Ji Chu Yi Xue Yu Lin Chuang Bian Ji Bu 33: 350-355, 2013 (In Chinese).
12. Tykvart J, Navrátil V, Sedlák F, Corey E, Colombatti M, Fracasso G, Koukolík F, Bařinka C, Š́cha P and Konvalinka J: Comparative analysis of monoclonal antibodies against prostate-specific membrane antigen (PSMA). Prostate 74: 1674-1690, 2014.

13. Osborne JR, Akhtar NH, Vallabhajosula S, Anand A, Deh K and Tagawa ST. Prostate-specific membrane antigen-based imaging. Urol Oncol 31: 144-154, 2013.

14. Holland JP, Divilov V, Bander NH, Smith-Jones PM, Larson SM, Lewis JS: 89Zr-DFO-J591 for immunoPET of prostate-specific membrane antigen in vivo. J Nucl Med 51: 1293-1300, 2010.

15. Kampmeier F, Williams JD, Maher J, Mullen GE and Blower PJ: Design and preclinical evaluation of a $99 \mathrm{mTc}$-labelled diabody of mAb J591 for SPECT imaging of prostate-specific membrane antigen (PSMA). EJNMMI Res 4: 13, 2014. 